\title{
Estudio longitudinal de estilos de vida en estudiantes de una universidad privada de Lima Metropolitana
}

\author{
Longitudinal study of lifestyles in students \\ of a private university of Metropolitan Lima
}

\author{
Cecilia Chau Pérez-Aranibarl y Mariela Tavera Palomino ${ }^{2}$
}

\begin{abstract}
Citación: Chau P.A., C. y Tavera P., M. (2020). Estudio longitudinal de estilos de vida en estudiantes de una universidad privada de Lima Metropolitana. Psicología y Salud, 30(2), 253-263. https:// doi.org/10.25009/pys.v30i2.2659.
\end{abstract}

\section{RESUMEN}

\begin{abstract}
El objetivo de la presente investigación fue comparar los estilos de vida en una muestra de estudiantes universitarios en dos periodos anuales. Para este propósito, se utilizó el cuestionario denominado Fantástico. Los resultados indican que hubo diferencias en estilos de vida tales como alimentación, percepción de manejo del estrés, consumo de cigarrillos y algunos comportamientos de seguridad. Se concluye que la mayoría de los estudiantes no mantenían estilos de vida adecuados en dichos periodos, aunque en el transcurso del tiempo algunos de ellos tendieron a mejorar.
\end{abstract}

Palabras claves: Estilos de vida; Estudiantes; Conductas saludables.

\begin{abstract}
The objective of the present study was to compare the lifestyles in a group of university students of two yearly periods. For this purpose, the "Fantastic" questionnaire was applied. The results revealed differences in such lifestyles as nutrition, perception of stress management, smoking, and security behavior. It is concluded that the majority of students in both periods did not maintain adequate lifestyles, although some of them tend, in time, to improve.
\end{abstract}

Key words: Lifestyles; Students; Health behavior.

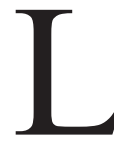

a etapa universitaria implica una serie de cambios emocionales, fisiológicos, ambientales, educativos, alimentarios y familiares que pueden asociarse a una serie de comportamientos de riesgo (Durán, Castillo y Vio, 2009; Gallardo, Muñoz, Planells y Lopez, 2015; Sabbah, Sabbah, Khamis, Sabbah y Droubi, 2013). Además, es una etapa de tránsito al mundo laboral (Intra, Roales y Moreno, 2011), por lo que se le considera como un puente entre la adolescencia y la edad adulta (Gonzalez, 2009).

Añadido a ello, la adolescencia y la juventud constituyen etapas vulnerables para el desarrollo de conductas de riesgo (Herrera, Wagner, Velasco, Borges y Lazcano, 2004; Kwan, Faulkner, Arbur-Nicitopoulos y Cairmey, 2013; Lucas, Santos, Pinheiro, Bianchini y Luiz, 2014; Roales, 2004). Los estudios coinciden en que la inactividad física, el bajo consumo de frutas y verduras, el consumo de alcohol (binge drinking) y de tabaco tienden a aumentar durante esta etapa (Da Franca y Colares, 2008; Kwan, Arbour-Nicitopoulos, Duru y Faulkner, 2016; Kwan, Cairney, Faulkner y Pullenayegum, 2012; Kwan, Faulkner, Arbur-Nicitopoulos y Cairmey 2013). Por todo ello, la población universitaria puede ser vulnerable a enfermar en el futuro (Intra, Roales y Moreno, 2011).

\footnotetext{
${ }^{1}$ Pontificia Universidad Católica del Perú, Av. Universitaria 1801 San Miguel, anexo 4597, 6262000 Lima, Perú. correo electrónico: cchau@pucp.edu.pe. Artículo recibido el 27 de marzo de 2019 y aceptado el 27 de febrero de 2020.

${ }^{2}$ Pontificia Universidad Católica del Perú, Almirante Manuel Villavicencio 1219, Dpto. 501, 997261014 Lince, Lima, Perú, correo electrónico: mariela.tavera@pucp.pe.
} 
A pesar de que el entorno universitario provee diversos conocimientos, la información existente y la comprensión que se tiene de los estilos de vida no necesariamente están relacionados con la práctica de hábitos saludables. En diversos estudios hechos con estudiantes de ciencias de la salud se ha encontrado que la información acerca de la salud y la nutrición no se relacionaba con prácticas saludables en quienes tenían acceso a la misma (Irazusta, Ruiz, Gil, Gil y Irazusta, 2005; Montero, Úbeda y García, 2006; Sajwani et al., 2009).

En general, los estudiantes universitarios tienden a calificar su salud como buena o muy buena a pesar de que reportan una baja ingestión de verduras y frutas, inconsistencia en la realización de la actividad física, falta de sueño y excesivo consumo de alcohol (American College Health Association [ACHA], 2015; Consorcio de Universidades de Lima, 2006; Durán et al., 2009; Lema et al., 2009; Meda et al., 2008; Saravia, 2013). En ese sentido, la percepción de salud no necesariamente es congruente con las enfermedades físicas o mentales que surgen en esta población, puesto que las personas pueden sentirse sanas y sin embargo no estarlo (Ardila, 2003).

En particular, la alimentación de un estudiante universitario no cumple con los requerimientos de una buena nutrición (Durá y Castroviejo, 2011; Vargas, Becerra y Prieto, 2008) debido a que se caracteriza por una ingesta inadecuada de calorías, tanto por defecto como por exceso (Irazustaet al., 2005); también la comida se elige en función del sabor, la disponibilidad y la conveniencia (Yahia, Achkar, Abdallag y Rizk, 2008). Además, los estudios reportan indicadores de sobrepeso y obesidad en esta población (Arroyo et al., 2006; Ve1la-Zarb y Elgar, 2009). En el Perú, en un estudio realizado por el Consorcio de Universidades de Lima (2006) con 1,256 estudiantes, se encontró que solamente $26.5 \%$ consumía frutas y verduras entre una o dos veces por semana. Asimismo, se halló que $22.8 \%$ de los estudiantes evaluados reportaron hábitos de alimentación no saludable.

En relación a la actividad física, las investigaciones hechas con jóvenes y adolescentes indican un bajo índice de actividad física, carencia que tiende a exacerbarse con el paso de los años (Buckworth y Nigg, 2004; Durá y Castroviejo, 2011; Durán et al., 2009; Kwan et al., 2012; Kwan et al.,
2013; Sigmundová, El Ansari, Sigmund y Frömel, 2011). El estudio del Consorcio de Universidades de Lima (2006) señaló una tendencia similar entre los estudiantes universitarios peruanos. Si bien $51.4 \%$ de los encuestados refirió practicar algún deporte, no cumplían necesariamente con los requisitos para ser considerada una práctica saludable. Becerra (2013) encontró en una muestra de 155 estudiantes de una universidad privada de Lima que los hombres realizaban más actividad física que las mujeres.

Por otro lado, en cuanto a la conducta de sueño, se ha encontrado que los estudiantes universitarios tienen una pobre calidad de sueño (Carter, Chopak-Foss y Punengwe, 2016; Lund, Reider, Whiting y Prichard, 2010), lo que se ha asociado con un estado de ánimo negativo y con problemas de salud mental, sobrepeso y obesidad (Concepción et al., 2014; Quick et al., 2015; Vargas, Flores y Robles, 2014) y con un bajo desempeño académico (Ahrberg, Dresler, Niedermaier, Steiger y Genzel, 2012; Gaultney, 2010; Gilbert y Weaver, 2010). En el Perú, se encontró que $14.1 \%$ de los estudiantes universitarios limeños no tenía una práctica saludable de sueño (Consorcio de Universidades de Lima, 2006), siendo la somnolencia una de las dificultades más reportadas por ellos (Boullosa, 2013). En la provincia de Lambayeque se halló que los estudiantes de medicina mostraban una pobre calidad del sueño (Del Piélago, Failoc, Plasencia y Díaz, 2013; Granados et al., 2013). Según la Secretaría Nacional de la Juventud (SENAJU) (2012), 56.4\% de los jóvenes de 15 a 29 años manifiestan cambios importantes en su ritmo de sueño, sobre todo las mujeres.

Diversos estudios reportan que el consumo de drogas legales e ilegales es un comportamiento de alto riesgo para la salud, e identifican su inicio en la adolescencia y juventud, lo que coincide con la etapa de los estudios universitarios (Centro de Información y Educación para la Prevención del Abuso de Drogas [CEDRO], 2005); Comisión Nacional para el Desarrollo y Vida sin Droga [DEVIDA], 2005; SENAJU, 2012).

En el caso específico de los estudiantes de este nivel, CEDRO (2005) informó que 97.7\% de ellos dijo haber consumido alcohol al menos una vez en su vida, y la Comunidad Andina (CAN, 2013) encontró en una muestra de 5,993 jóvenes que $71.7 \%$ de ellos había ingerido alcohol en el último año. Tal 
estudio advierte que $18.23 \%$ tendría un consumo riesgoso o perjudicial y que $10.54 \%$ podría desarrollar dependencia a esta bebida.

Otra sustancia altamente prevalente en la población universitaria es el tabaco. Si bien en los últimos años su consumo ha ido disminuyendo, el porcentaje de la población universitaria es mayor que el de la población general (CAN, 2013). Por otro lado, se ha encontrado que $11.78 \%$ de estos jóvenes habría probado marihuana al menos una vez en su vida (CAN, 2013). Si bien el porcentaje podría considerarse bajo en relación al consumo de alcohol o tabaco, es sin embargo mayor al reportado por la población de 12 a 65 años (3.8\%) (DEVIDA, 2005) y de 19 a 24 años (10.5\%) (CEDRO, 2005).

La organización del tiempo libre es un estilo de vida que afecta la elección de comportamientos que el sujeto lleva a cabo con frecuencia (Rodríguez y Agulló, 1999). Los estudios indican que los estudiantes que tienen un adecuado manejo del tiempo tienen igualmente mayores niveles de satisfacción con la vida (Akgül, Faith y Karaküçük, 2016; Wang, Kao, Huan y Wu, 2011). En el Perú, Saravia (2013) encontró en un grupo de 448 estudiantes universitarios limeños de entre 18 y 29 años que la organización del tiempo libre y del sueño eran sólidos predictores de su salud mental y física, lo que quiere decir que aquellos jóvenes que tenían más estructurado su tiempo libre y su sueño tendían a presentar mejores niveles de salud mental.

Se puede señalar entonces que la población universitaria manifiesta y mantiene por lo general estilos de vida inadecuados que podrían generar diversos problemas físicos y mentales. Es así que la universidad, como una institución educativa que alberga una cantidad importante de adolescentes y jóvenes, es un espacio ideal para promover su salud y bienestar (Consorcio de Universidades de Lima, 2013; Kühne y Pérez, 2011; Lange y Vio, 2006).

En este marco, se considera relevante efectuar investigaciones que permitan analizar de forma general los estilos de vida y los cambios que la población universitaria experimenta a través del tiempo, con la finalidad de fomentar acciones que faciliten intervenciones en el corto, mediano y largo plazo en favor del fomento de una cultura de salud. En ese sentido, el objetivo del presen- te estudio fue describir los estilos de vida de una muestra de estudiantes universitarios limeños y su variación a través del tiempo.

\section{MÉTODO}

\section{Participantes}

El presente estudio tuvo dos momentos. El primero se llevó a cabo en el año 2012 y el segundo en 2014. Los participantes cursaban los primeros años de estudio y pertenecían a las siguientes facultades de una universidad privada en Lima: Letras y Ciencias Humanas, Derecho, Ciencias e Ingeniería, Ciencias y Artes de la Comunicación, Ciencias Sociales, Artes, Gestión y Alta Dirección, Arquitectura y Urbanismo y Educación. El muestreo se realizó incluyendo a estudiantes de todas las facultades, hombres y mujeres, y de todas las escalas de pago. Se calculó el número necesario de participantes de cada facultad en función del número total de alumnos inscritos en ellas. Se enviaron las invitaciones en tres ocasiones, pero no todas las facultades cumplieron con la cuota necesaria. El cálculo se realizó a través de la Dirección de Informática de la institución.

El primer estudio contó con la colaboración de 683 alumnos (68\% de ellos nacidos en la ciudad de Lima), cuyas edades fluctuaron entre $16 \mathrm{y}$ 22 años $(M=19.16$; D.E. $=1.63)$; del total, 57.5\% fueron mujeres y $42.5 \%$ hombres.

La mayoría de estudiantes pertenecía al área de Estudios Generales (Letras: 34.6\%; Ciencias: $20.5 \%$ ), seguido de Ciencias e Ingeniería (13.2\%) y otras facultades (que en conjunto ascendieron a $31.8 \%$ ). De ellos, 92.1\% no estaba becado. Hubo un total de $.9 \%$ de datos perdidos.

En el segundo estudio, la muestra estuvo conformada por 218 jóvenes que ya habían participado en el primer estudio. La edad de los participantes en esta medición osciló entre los 19 y 24 años de edad ( $\mathrm{M}=21.28$, D.E. $=1.56)$, de ambos sexos, 72 hombres (33\%) y 146 mujeres (67\%). De ellos, $74.3 \%$ eran originarios de Lima.

$\mathrm{Al}$ igual que en el primer momento, más de la mitad de los participantes se hallaba en el área de Estudios Generales (40.8\% hombres y 16.1\% mujeres), $10.1 \%$ en Ciencias e Ingeniería y el resto en otras facultades; $93.1 \%$ no estaba becado. 
Cabe mencionar que se obtuvo una mayor tasa de respuesta por parte de las mujeres en ambas mediciones $(M=67 \%, H=33 \%)$. Asimismo, el número de hombres y mujeres que no participaron en la segunda medición fue similar $(\mathrm{H}=48.2 \% \mathrm{y}$ $\mathrm{M}=51.8 \%$ ).

La aplicación del cuestionario se efectuó de manera virtual. Se informó a los participantes las condiciones del estudio mediante un formato de consentimiento informado sobre el propósito y la naturaleza del estudio, el carácter voluntario de la participación y la confidencialidad de datos que se obtuvieran.

\section{Instrumento}

\section{Cuestionario Estilos de Vida (FANTÁSTICO)}

(Wilson y Ciliska, 1984).

Este cuestionario incluye componentes de salud física, psicológica y social. Si bien el instrumento original consta de 25 enunciados, en el presente estudio se utilizó la versión de 30 ítems, los cuales se agrupan en los siguientes dominios: asociatividad, actividad física, nutrición, familia y amigos, tabaco, alcohol y otras drogas, sueño, estrés, trabajo, tipo de personalidad, introspección, control de salud, conducta sexual y otro tipo de prácticas. Cada uno de los enunciados cuenta con tres opciones de respuesta, con un valor numérico de 0 a 2 para cada categoría, y que se califican mediante una escala tipo Likert con calificaciones de 0 a 100 puntos.

Este instrumento reporta adecuados índices de confiabilidad (coeficientes $\alpha$ de Cronbach de entre .50 y .89), en estudios realizados en Chile (Cancino, Urzúa y Ulloa, 2009), Colombia (Bohorquez, Briceño y Mora, 2012) y Perú (Honorio, 2010). En la presente investigación se obtuvieron coeficientes de .62 a .79 .

\section{Procedimiento y análisis de datos}

El estudio contó de la colaboración de la Dirección de Informática de la institución evaluada, la cual se encargó de la realización del muestreo y la aplicación de los instrumentos de manera virtual. La primera aplicación inició el mes de octubre 2012 y duró dos semanas. Se otorgaron dos semanas adicionales enviando una alerta más de parti- cipación a los alumnos seleccionados. La segunda aplicación se realizó dos años más tarde, en los mismos tiempos del año y a los mismos estudiantes, obteniéndose una tasa de respuesta menor.

Se utilizó el software estadístico IBM SPSS Statistics, v. 22, para el análisis de los datos. Se realizó un análisis comparativo de tipo descriptivo de las variables sociodemográficas de sexo y edad en el grupo que participó en ambas mediciones, para lo cual se utilizó la prueba $T$ de Wilcoxon para muestras relacionadas.

\section{RESULTADOS}

Los resultados hallados muestran que, de los treinta ítems del Cuestionario FANTÁSTICO, solo siete de ellos tuvieron diferencias significativas $(p<.05)$ entre la medición de 2012 y la de 2014. Estos ítems pertenecían a los dominios Familia y amigos, Nutrición, Tabaco, Tipo de personalidad y Otras conductas. No se hallaron diferencias significativas en ninguno de los ítems de los dominios Asociación y actividad física, Alcohol y otras drogas, Sueño y estrés y Control de salud y conducta sexual.

En relación al dominio de Nutrición, se apreció un cambio significativo en dos ítems. En el primero de ellos, "A menudo consumo mucha azúcar o sal o comida chatarra o con mucha grasa" $(p<.05 ; \mathrm{Z}=-3.29)$, se observa que la opción de respuesta "Todas estas" decrece en 3.2\%, mientras que las respuestas "Ninguna de estas" y "Algunas de estas" aumentaron en 3.2 y $2.3 \%$, respectivamente (Tabla 1 ).

Tabla 1. Ítem "A menudo consumo mucha azúcar o sal o comida chatarra o con mucha grasa".

\begin{tabular}{|c|c|c|c|c|}
\hline \multirow{2}{*}{$\begin{array}{l}\text { Opción } \\
\text { de respuesta }\end{array}$} & \multicolumn{2}{|c|}{$\begin{array}{c}2012 \\
(N=218)\end{array}$} & \multicolumn{2}{|c|}{$\begin{array}{c}2014 \\
(N=218)\end{array}$} \\
\hline & $\%$ & $n$ & $\%$ & $n$ \\
\hline Ninguna de estas & 17.4 & 38 & 20.6 & 45 \\
\hline Algunas de estas & 58.3 & 127 & 60.6 & 132 \\
\hline Todas estas & 14.7 & 32 & 11.5 & 25 \\
\hline Casos perdidos & 9.6 & 21 & 7.3 & 16 \\
\hline
\end{tabular}

En el dominio Tabaco, dos ítems obtuvieron diferencias significativas entre ambas mediciones. 
Uno de ellos fue "Yo fumo cigarrillos" $(p<.05$; $Z=-2.52)$. Se observa que la mayoría de alumnos, 54.6 y $54.1 \%$, respectivamente, marcaron la opción "No en los últimos cinco años" y "No en el último año", siendo esta última opción de respuesta la de mayor variación, presentando un aumento de 5\% (Tabla 2).

Tabla 2. "Yo fumo cigarrillos".

\begin{tabular}{|l|r|c|r|r|}
\hline \multirow{2}{*}{$\begin{array}{c}\text { Opción } \\
\text { de respuesta }\end{array}$} & \multicolumn{2}{c|}{$\begin{array}{c}\text { 2012 } \\
(\boldsymbol{N}=\mathbf{2 1 8})\end{array}$} & \multicolumn{2}{c|}{$\begin{array}{c}\text { 2014 } \\
(\boldsymbol{N}=\mathbf{2 1 8})\end{array}$} \\
\cline { 2 - 5 } & \multicolumn{1}{c|}{$\boldsymbol{c}$ \% } & \multicolumn{1}{c|}{$\boldsymbol{n}$} & \multicolumn{1}{c|}{$\%$} & $\boldsymbol{n}$ \\
\hline No en los últimos cinco años & 54.6 & 119 & 54.1 & 118 \\
\hline No en el último año & 7.8 & 17 & 12.8 & 28 \\
\hline Sí este año & 23.4 & 51 & 22.5 & 49 \\
\hline Casos perdidos & 14.2 & 31 & 10.6 & 23 \\
\hline
\end{tabular}

$* p<.05(Z=-2.524)$.

El otro ítem del dominio Tabaco fue "Generalmente fumo $[n]$ cigarrillos por día” ( $p<0.05 ; Z=3.75)$. La mayoría de alumnos no fumaba ningún cigarrillo al día en las dos mediciones (2012: 69.7\%; 2014: 75.7\%). Asimismo, hubo un aumento de 6\% en la segunda medición (Tabla 3).

Tabla 3. "Generalmente fumo [n] cigarrillos por día".

\begin{tabular}{|l|r|c|r|r|}
\hline \multirow{2}{*}{$\begin{array}{c}\text { Opción } \\
\text { de respuesta }\end{array}$} & \multicolumn{2}{c|}{$\begin{array}{c}\mathbf{2 0 1 2} \\
(N=\mathbf{2 1 8})\end{array}$} & \multicolumn{2}{c|}{$\begin{array}{c}\mathbf{2 0 1 4} \\
(N=\mathbf{2 1 8})\end{array}$} \\
\cline { 2 - 5 } & $\mathbf{\%}$ & \multicolumn{1}{c|}{$\boldsymbol{n}$} & \multicolumn{1}{c|}{$\%$} & \multicolumn{1}{c|}{$\boldsymbol{n}$} \\
\hline Ninguno & 69.7 & 152 & 75.7 & 165 \\
\hline De 0 a 10 & 18.4 & 40 & 15.5 & 34 \\
\hline Más de 10 & 0 & 0 & 0.5 & 1 \\
\hline Casos perdidos & 11.9 & 26 & 8.3 & 18 \\
\hline$* * * p<001(Z=-3.754)$ &
\end{tabular}

En relación al dominio Trabajo y tipo de personalidad, tres de sus ítems obtuvieron diferencias significativas. Uno de ellos fue "Parece que ando acelerado(a)" ( $p<0.05 ; Z=-3.54)$. En este se aprecia que, en ambas mediciones, la mayoría de alumnos: $58.7 \%$ en 2012 y $59.1 \%$ en 2014 , marcaron la opción "Algunas veces" (Tabla 4).

Sobre el dominio de Introspección, en el ítem "Me siento tenso(a) o apretado(a)" ( $p<0.05$; $Z=-3.38)$ se muestra un considerable aumento de la opción "Algunas veces" (12.5\%). Así también es la que obtuvo mayor tasa de respuesta en las dos mediciones (Tabla 5).
Tabla 4. "Parece que ando acelerado(a)".

\begin{tabular}{|c|c|c|c|c|}
\hline \multirow{2}{*}{$\begin{array}{c}\text { Opción } \\
\text { de respuesta }\end{array}$} & \multicolumn{2}{|c|}{$\begin{array}{c}2012 \\
(N=218)\end{array}$} & \multicolumn{2}{|c|}{$\begin{array}{c}2014 \\
(N=218)\end{array}$} \\
\hline & $\%$ & $n$ & $\%$ & $n$ \\
\hline Casi nunca & 9.1 & 20 & 11.5 & 25 \\
\hline Algunas veces & 58.7 & 128 & 59.1 & 129 \\
\hline A menudo & 21.6 & 47 & 21.1 & 46 \\
\hline Casos perdidos & 10.6 & 23 & 8.3 & 18 \\
\hline
\end{tabular}

Tabla 5. "Me siento tenso(a) o apretado(a)".

\begin{tabular}{|l|r|r|r|r|}
\hline \multirow{2}{*}{$\begin{array}{c}\text { Opción } \\
\text { de respuesta }\end{array}$} & \multicolumn{2}{|c|}{$\begin{array}{c}\mathbf{2 0 1 2} \\
(N=\mathbf{2 1 8})\end{array}$} & \multicolumn{2}{c|}{$\begin{array}{c}\text { 2014 } \\
(N=218)\end{array}$} \\
\cline { 2 - 5 } & $\mathbf{\%}$ & \multicolumn{1}{c|}{$\boldsymbol{n}$} & \multicolumn{1}{c|}{$\%$} & \multicolumn{1}{c|}{$\boldsymbol{n}$} \\
\hline Casi nunca & 9.6 & 21 & 7.3 & 16 \\
\hline Algunas veces & 53.2 & 116 & 65.7 & 143 \\
\hline A menudo & 27.1 & 59 & 19.7 & 43 \\
\hline Casos perdidos & 10.1 & 22 & 7.3 & 16 \\
\hline
\end{tabular}

Finalmente, en el dominio Otras conductas, el enunciado "Como peatón, pasajero del transporte público y/o automovilista, soy respetuoso(a) de las ordenanzas de tránsito" ( $p<0.05 ; Z=-2.61)$ se muestra que en ambas mediciones la mayoría de alumnos marcaron la opción "Siempre": 59.6 y $62.4 \%$, respectivamente (Tabla 6 ).

Tabla 6. "Como peatón, pasajero del transporte público o automovilista, soy respetuoso(a) de las ordenanzas del tránsito".

\begin{tabular}{|c|c|c|c|c|}
\hline \multirow{2}{*}{$\begin{array}{c}\text { Opción } \\
\text { de respuesta }\end{array}$} & \multicolumn{2}{|c|}{$\begin{array}{c}2012 \\
(N=218)\end{array}$} & \multicolumn{2}{|c|}{$\begin{array}{c}2014 \\
(N=218)\end{array}$} \\
\hline & $\%$ & $n$ & $\%$ & $n$ \\
\hline Casi nunca & 0 & 0 & 1.4 & 3 \\
\hline A veces & 29.4 & 64 & 29.4 & 64 \\
\hline Siempre & 59.6 & 130 & 62.4 & 136 \\
\hline Casos perdidos & 11 & 24 & 6.8 & 15 \\
\hline
\end{tabular}

Por último, respecto al ítem "Uso cinturón de seguridad" ( $p<0.05 ; Z=-2.05)$, en 2014 se muestra una mayor frecuencia en la respuesta "Siempre" $(7.3 \%)$ y un descenso en las opciones "Casi nunca" (1.3\%) y "A veces" (2.7\%). Así también, se observa que la opción "Siempre" fue la que obtuvo la mayor tasa de respuesta en ambas mediciones (Tabla 7). 
Tabla 7. "Uso cinturón de seguridad".

\begin{tabular}{l|l|r|r|r|}
\hline \multirow{2}{*}{$\begin{array}{c}\text { Opción } \\
\text { de respuesta }\end{array}$} & \multicolumn{2}{c|}{$\begin{array}{c}\mathbf{2 0 1 2} \\
(\boldsymbol{N}=\mathbf{2 1 8})\end{array}$} & \multicolumn{2}{c|}{$\begin{array}{c}\mathbf{2 0 1 4} \\
(\boldsymbol{N}=\mathbf{2 1 8})\end{array}$} \\
\cline { 2 - 5 } & \% & \multicolumn{1}{c|}{$\boldsymbol{n}$} & \multicolumn{1}{c|}{$\%$} & \multicolumn{1}{c|}{$\boldsymbol{n}$} \\
\hline Casi nunca & 1.8 & 4 & 0.5 & 1 \\
\hline A veces & 13.3 & 29 & 10.6 & 23 \\
\hline Siempre & 73.4 & 160 & 80.7 & 176 \\
\hline Casos perdidos & 11.5 & 25 & 8.2 & 18 \\
\hline & $* p<.05(Z=-2.045)$.
\end{tabular}

\section{DISCUSIÓN}

La adolescencia y juventud constituyen periodos clave para la adopción de comportamientos relacionados con la salud, entre los que destacan la inactividad física, el consumo de alimentos considerados no saludables, el uso del tabaco, alcohol y otras drogas, las prácticas sexuales de riesgo y las conductas violentas o imprudentes que ponen en riesgo su vida, entre otros (Berger, 2001; Santrock, 2006).

Los estudios muestran que los jóvenes no perciben riesgos en relación a las consecuencias de no tener un estilo de vida saludable, pues se encuentran escasamente identificados con los problemas de salud, los que suelen aparecer a edades más tardías (Santrock, 2006; Taylor, 2007). Por ello, se hace necesaria la focalización en el conocimiento y la evaluación de las conductas de salud en la población joven, al igual que de los factores psicológicos que se asocian a ella.

Los hallazgos indican que, de los dominios del cuestionario FANTÁSTICO, siete de ellos mostraron diferencias significativas entre los periodos 2012 y 2014.

En cuanto a la nutrición, se observa que los participantes disminuyeron el consumo de altos contenidos de azúcar, sal o grasas, lo que sugiere que tienen ahora una alimentación más sana. Este hallazgo plantea la relevancia del entorno como facilitador de conductas que tendrán un efecto en la salud (Dooris, 2005). En el ámbito universitario, y en general en la sociedad, ha aumentado la disponibilidad de alimentos saludables y hay ya una mayor conciencia de la importancia de su consumo (Andonova, 2014; Freedman y Connors, 2010). De la misma manera, en los entornos educativos hay esfuerzos por facilitar a los estudiantes cono- cimientos sobre la nutrición, lo que posiblemente podría relacionarse con el creciente consumo de alimentos saludables (Ha y Caine-Bish, 2009; Ke1ly, Mazzeo y Bean, 2013; Yahia, Brown, Rapley y Chung, 2016).

De modo similar, una adecuada dieta se relaciona con el control de peso. Esta relación adquiere la mayor importancia durante la adolescencia y la juventud debido a la valoración social que tiene el peso y la forma del cuerpo (Hidalgo, Hidalgo, Rasmussen y Montaño, 2011; Saravia, 2013). A ello se suma la constante exposición de cuerpos esbeltos en los medios de comunicación, que podrían generar cambios en los patrones de alimentación de los jóvenes (Knobloch-westerwick y Crane, 2011; Lattimore y Hutchinson, 2010; Thomsen, 2002).

En relación al consumo de tabaco, se encontró que los jóvenes universitarios consumen una menor cantidad de cigarrillos, e incluso algunos de ellos no han consumido ninguno durante el último año. Esta tendencia es global y responde a las medidas para detener el tabaquismo sugeridas por la Organización Mundial de la Salud (OMS) (2013). Entre estas medidas se encuentran la advertencia de los peligros del tabaco, la prohibición de su publicidad, promoción y patrocinio, y el aumento del impuesto de esta sustancia (OMS, 2008). En el Perú ha habido una mayor regulación del tabaco mediante la ley que prohibe fumar en entidades educativas y públicas cerradas (E1 Peruano, 2010; Ministerio de Salud [MINSA], 2016). Es preciso destacar entonces la importancia del entorno debido a que desempeñará un papel fundamental para el desarrollo o creación hábitos de salud (Lange y Vio, 2006). En ese sentido, la disponibilidad o no de sustancias dañinas en ese entorno es un factor importante en su consumo (Oficina de las Naciones Unidades Contra la Droga y el Delito [UNODC], 2013).

A pesar de los datos alentadores sobre la disminución del consumo de tabaco en todo el mundo, y específicamente en la población universitaria, la CAN (2013), en un estudio realizado con estudiantes universitarios de Bolivia, Colombia, Ecuador y Perú, advierte que la prevalencia del consumo de tabaco había aumentado de $31.1 \%$ en el año 2009 a 33.7\% en 2012. Este hallazgo indica la importancia de conocer las características particulares de esta población con la finalidad de 
diseñar programas y medidas de promoción eficaces que fortalezcan la concienciación sobre los efectos nocivos de esta droga lícita.

En relación al dominio de Tipo de personalidad, se halló un aumento de 53.2 a $65.7 \%$ en cuanto a la percepción del estrés en la opción "Alguna vez", mientras que el porcentaje de estudiantes que señalaron sentirse tensos "A menudo" disminuyó de 27.1 a 19.7\%. Lo anterior podría sugerir que la tensión percibida por los estudiantes universitarios surge durante ciertos periodos; es decir, que no se trata de una condición permanente o crónica. Al respecto, las investigaciones indican que el nivel de estrés durante el periodo de las evaluaciones o exámenes tiende a aumentar de manera significativa (Monzón, 2007; Pozos, Preciado, Acosta, Aguilera y Delgado 2014). Tal resultado muestra que los estudiantes consideran los exámenes, la sobrecarga de trabajos y la falta de tiempo, entre otros factores, como los principales estresores de la vida universitaria (Boullosa, 2013; Pozos et al., 2014). De la misma manera, es importante considerar que a lo largo del tiempo los jóvenes desarrollan estrategias que les permiten lidiar con dicho estrés (Polo, Hernández y Poza, 1996). Según indican los estudios realizados con estudiantes peruanos, utilizan en mayor medida las estrategias orientadas al problema (Becerra, 2013; Boullosa, 2013; Cassaretto, Chau, Oblitas y Valdez, 2003).

Por último, en relación a Otras conductas, los estudiantes reportan ser más respetuosos como peatones, pasajeros o conductores de las normas de seguridad vial. Este mayor acatamiento de las normas podría relacionarse con la formación ciudadana propia del espíritu de la educación superior, que permite reconocer al otro en igualdad de derechos y condiciones (Martínez, 2006), lo que repercute no únicamente en lo concerniente a la ciudadanía y las relaciones interpersonales, sino también en el cuidado físico de lo que rodea al individuo. Posiblemente otro factor interviniente podría ser el énfasis y el esfuerzo por parte de las unidades policiales para lograr el cumplimiento de las normas de tránsito. En el Perú, durante los últimos seis años, la reforma de transporte ha incluido la creación de la Superintendencia de Transporte Terrestre de Personas, Carga y Mercancía (cfr. SUTRAN, 2011) que ha puesto énfasis en el desarrollo de proyectos de prevención vial (El Peruano,
2009; Ministerio de Transportes y Comunicaciones, $\mathrm{s} / \mathrm{f}$ ). De la misma manera, se ha formulado el Plan Nacional de Seguridad Vial del Perú, en el cual esta es considerada como prioridad de la gestión pública y en la que la educación vial desempeña un importante rol para su ejecución (Ministerio de Transportes y Comunicaciones, 2015).

En cuanto a las limitaciones del presente estudio, se podría señalar la importante disminución del número de participantes en el segundo momento. Inicialmente se contó con la colaboración de 683 estudiantes en 2012, pero solo de 218 en 2014. Por otro lado, se debe tomar en cuenta que el instrumento recoge solo algunos estilos de vida; además, representa una primera aproximación al estudio del problema analizado porque no brinda información más precisa y detallada sobre la complejidad de esas conductas. Se debe también tomar en cuenta el efecto de la deseabilidad social, por lo cual se pudo haber registrado algunos estilos de vida inadecuados, no obstante se garantizó a los participantes el anonimato y la confidencialidad de sus datos.

Se recomienda fomentar la ejecución de estudios longitudinales que hagan posible identificar los cambios en los estilos de vida que aparecen a lo largo del tiempo en los espacios universitarios. De la misma manera, resultaría enriquecedor estudiar cada estilo de vida, considerando no solo su práctica, sino también los factores que facilitan o dificultan que se lleven a cabo determinadas conductas de salud. En esa línea, sería trascendente complementar estudios descriptivos con enfoques cualitativos que permitan una profundización en las dinámicas de tales conductas.

Los resultados de la presente investigación representan un marco general de la mejora o deterioro de las conductas de salud, lo cual podría ser la base para sustentar y crear programas de intervención para la promoción de estilos de vida saludables. De esta manera, se podría comenzar a trabajar con los estilos que se encuentren más debilitados, así como también continuar fortaleciendo aquellos que muestran mejoras significativas.

Como conclusión general, se considera que los estilos de vida de los estudiantes universitarios manifestaron ligeras mejoras tanto en el año 2012 como en el 2014. No obstante, no hay que perder de vista que en el presente estudio se consideraron 
solamente algunas conductas, por lo cual no se desestima que exhiban comportamientos de riesgos que afecten su salud. Al tener en cuenta que las características propias de su etapa de desarrollo impulsan frecuentemente comportamientos de riesgo, se considera que una de las tareas de la universidad, además de fomentar la educación para la salud, es hacer menos disponibles aquellos estímulos que propicien conductas no saludables.

\section{REFERENCIAS}

Ahrberg, K., Dresler, M., Niedermaier, S., Steiger, A. y Genzel, L. (2012). The interaction between sleep quality and academic performance. Journal of Psychiatric Research, 46(12), 1618-1622. doi: 10.1016/j.jpsychires.2012.09.008.

Akgül, B., Fatih, I. y Karaküçük, S. (2016). Evaluating free time management and its relation to life satisfaction, most participated leisure activities and gender among college students in Turkey. Social Sciences and Humanities Journal, 11(2), 2-18.

American College Health Association (ACHA) (2015). American College Health Association-National College Health Assessment II: Reference Group Executive Summary Spring 2015. Hanover, MD: American College Health Association.

Andonova, A. (2014). Information and awareness of the students' healthy eating. Trakia Journal of Sciences, 12(1), 362-366.

Ardila, R. (2003). Calidad de vida: una definición integradora. Revista Latinoamericana de Psicología, 35(2), 161-164.

Arroyo, I., Rocandio, A.M., Ansotegui, L., Pascual, E., Salces, I. y Rebato, E. (2006). Calidad de la dieta, sobrepeso y obesidad en estudiantes universitarios. Nutrición Hospitalaria, 21(6), 673-679.

Becerra, S. (2013). Rol de estrés percibido y su afrontamiento en las conductas de salud de estudiantes universitarios de Lima. Tesis inédita de Maestría. Lima: Pontificia Universidad Católica del Perú.

Berger, K. (2001). Psicología del desarrollo: adultez y vejez. Madrid: Médica Panamericana, S.A.

Bohorquez, L., Briceño, C. y Mora, L. (2012). Caracterización de los estilos de vida del adulto mayor que asiste al Coliseo de la Luna en Chía. Trabajo de grado. Chía (Colombia): Universidad de la Sabana.

Boullosa, G. (2013). Estrés académico y afrontamiento en un grupo de estudiantes de una universidad privada de Lima. Tesis inédita de licenciatura. Lima: Pontificia Universidad Católica del Perú.

Buckworth, J. y Nigg, C. (2004). Physical activity, exercise, and sedentary behavior in college students. Journal of American College Health, 53(1), 28-34.

Cancino, J., Urzúa, R. y Ulloa, D. (2009). Estilos de vida y nivel de actividad física de los alumnos de kinesiología de la Universidad de las Américas (Chile) durante el primer semestre del año 2008. Kronos, 8(16), 39-44.

Carter, B., Chopak-Foss, J. y Punengwe, F. (2016). An analysis of the sleep quality of undergraduate students. College Student Journal, 50(3), 315-322.

Cassaretto, M., Chau, C., Oblitas, H. y Valdez, N. (2003). Estrés y afrontamiento en estudiantes de psicología. Revista de Psicología de la PUCP, 21(3), 363-392.

Centro de Información y Educación para la Prevención del Abuso de Drogas (CEDRO) (2005). Estudio sobre drogas en universitarios de Lima. Lima: CEDRO.

Comisión Nacional para el Desarrollo y Vida sin Drogas (DEVIDA) (2005). I Encuesta sobre información, hábitos y actitudes hacia el consumo de sustancias psicoactivas en universitarios de Lima metropolitana, 2005. Lima: Mesa de Trabajo de Universidades/DEVIDA.

Comunidad Andina (CAN) (2013). II Estudio epidemiológico andino sobre consumo de drogas en la población universitaria. Informe Perú, 2012. Secretaría General de la Comunidad Andina.

Concepción, T., Barbosa, C., Vélez, J.C., Peper, M., Andrade, A. Yanez, D. y Williams, M. (2014). Daytime sleepiness, poor sleep quality, eveningness chronotype, and common mental disorders among Chilean college students. Journal of American College Health, 62(7), 441-448. doi: 10.1080/07448481.2014.917652.

Consorcio de Universidades de Lima (2006). Perfil de los estudiantes del Consorcio de Universidades: resumen y análisis. Lima: Autor.

Consorcio de Universidades de Lima (2013). Guía para universidades saludables. Lima: Autor.

Da Franca, C. y Colares, V. (2008). Comparative study of health behavior among college students at the start and end of their courses. Revista de Saúde Pública, 42(3), 420-427.

Del Piélago, A.F., Failoc, V.E., Plasencia, E.A. y Díaz, C. (2013). Calidad de sueño y estilo de aprendizaje en estudiantes de Medicina Humana de la Universidad Nacional Pedro Ruiz Gallo. Acta Médica Peruana, 30(4), 63-68.

Dooris, M. (2005). Healthy settings: challenges to generating evidence of effectiveness. Health Promotion International, 21(1), 55-65. doi: doi:10.1093/heapro/dai030. 
Durá, T. y Castroviejo, A. (2011). Adherencia a la dieta mediterránea en la población universitaria. Nutrición Hospitalaria, 26(3), 602-608.

Durán, S., Castillo, M. y Vio, F. (2009). Diferencias en la calidad de vida de estudiantes universitarios de diferente año de ingreso del campus Antumapu. Revista Chilena de Nutrición, 36(3), 200-209.

El Peruano (2009, 16 de junio). Normas Legales 397625. Lima. Recuperado de http://www.gacetajuridica.com.pe/servicios/normaspdf_2009/junio/16-06-2009.pdf.

El Peruano (2010, 1 de abril). Normas Legales 29517. Lima. Recuperado de http://www.inen.sld.pe/portal/documentos/pdf/educacion/24092013_LEY_29517.pdf.

Freedman, M.R. y Connors, R. (2010). Point-of-purchase nutrition information influences food-purchasing behaviors of college students: A pilot study. Journal of the American Dietetic Association, 110, 1222-1226. doi: 10.1016/j.jada.2010.05.002.

Gallardo E., A., Muñoz, M.J., Planells, E.M. y López, I. (2015). La etapa universitaria no favorece el estilo de vida saludable en las estudiantes granadinas. Nutrición Hospitalaria, 31(2), 975-979. doi: 10.3305/nh.2015.31.2.8303.

Gaultney, J.F. (2010). The prevalence of sleep disorders in college students: impact on academic performance. Journal of American College Health, 59(2), 91-97. doi: 10.1080/07448481.2010.483708.

Gilbert, S.P. y Weaver, C.C. (2010). Sleep quality and academic performance in university students: a wake-up call. Journal of College Student Psychotherapy, 24, 295-306. doi: 10.1080/87568225.2010.509245.

Granados C., Z., Bartra A., A., Bendezú B., D., Huamanchumo M., J., Hurtado N., E., Jiménez F., J. y Chang D., D. (2013). Calidad del sueño en una facultad de medicina de Lambayeque. Anales de la Facultad de Medicina, 74(4), 311-314.

Ha, E. y Caine-Bish, N. (2009). Effect of nutrition intervention using a general nutrition course for promoting fruit and vegetable consumption among college students. Journal of Nutrition Education and Behavior, 41(2), 103-109. doi: 10.1016/j. jneb.2008.07.001.

Herrera, M., Wagner, F., Velasco, E., Borges G. y Lazcano, E. (2004). Inicio en el consumo de alcohol y tabaco y transición a otras drogas en estudiantes de Morelos, México. Salud Pública de México, 46, 132-140.

Hidalgo S.M., A., Hidalgo R., C., Rasmussen C., B. y Montaño E., R. (2011). Calidad de vida según percepción y comportamientos de control de peso por género, en estudiantes universitarios adolescentes en México. Cadernos de Saúde Pública. 27(1), 67-77.

Intra, M., Roales N., J. y Moreno, E. (2011). Cambio en las conductas de riesgo y salud en estudiantes universitarios argentinos a lo largo del período educativo. International Journal of Psychology and Psychological Therapy, 11(1), 139-147.

Irazusta, A., Ruiz, F., Gil, S., Gil, G. e Irazusta, J. (2005). Hábitos de vida de los estudiantes de enfermería. Zainak. Cuadernos de Antropología-Etnografía, 27, 99-107.

Kelly, N.R., Mazzeo, S.E. y Bean, M.K. (2013). Systematic review of dietary interventions with college students: directions for future research and practice. Journal of Nutrition Education and Behavior, 45(4), 304-313. doi: 10.1016/j.jneb.2012.10.012.

Knobloch-westerwick, S. y Crane, J. (2011). A losing battle: effects of prolonged exposure to thin-ideal images on dieting and body satisfaction. Communication Research, 39(1), 79-102.

Kühne, W. y Pérez, P. (2011). Guía de apoyo psicológico para universitarios: formación integral y autocuidado (2a ed.). Santiago de Chile: Universidad de Santiago de Chile.

Kwan, M.Y., Arbur-Nicitopoulos, K.P., Duku, E. y Faulkner, G.E. (2016). Patterns of multiple health risk-behaviours in university students and their association with mental health: application of latent class analysis. Research, Policy and Practice, 36(8), 163-170.

Kwan, M.Y., Cairney, J., Faulkner, G.E. y Pullenayegum, E.E. (2012). Physical activity and other health-risk behaviors during the transition into early adulthood. A longitudinal cohort study. American Journal of Preventive Medicine, 42(1), 14-20. doi: 10.1016/j.amepre.2011.08.026.

Kwan, M.Y., Faulkner, G.E., Arbur-Nicitopoulos, K.P. y Cairmey, J. (2013). Prevalence of health-risk behaviours among Canadian post-secondary students: descriptive results from the National College Health Assessment. BMC Public Health, 13(1), 1-6. doi: 10.1186/1471-2458-13-548.

Lange, I. y Vio, F. (2006). Guía para universidades saludables y otras instituciones de educación superior. Santiago de Chile: Ministerio de Salud.

Lattimore, P. y Hutchinson, R. (2010). Perceived calorie intake and state body-image satisfaction in women attempting weight loss: A preliminary investigation. Body Image, 7(1), 15-21.

Lema, L., Salazar, I., Varela, M., Tamayo, J., Rubio, A. y Botero, A. (2009). Comportamiento y salud de los jóvenes universitarios: satisfacción con el estilo de vida. Pensamiento Psicológico, 5(12), 71-88.

Lucas P., R., Santos S., D.A., Pinheiro G., A., Bianchini Q., T.M. y Luiz P., E. (2014). Sociodemographic determinants of university student's lifestyles. Revista de Salud Pública, 16(3), 382-393. doi: https://doi.org/10.15446/rsap.v16n3.33164.

Lund, H.G., Reider, B.D., Whiting, A.B. y Prichard, J.R. (2010). Sleep patterns and predictors of disturbed sleep in a large population of college students. Journal of Adolescent Health, 46(2), 124-132. doi: 10.1016/j.jadohealth.2009.06.016. 
Martínez, M. (2006). Formación para la ciudadanía y educación superior. Barcelona: Universidad de Barcelona.

Meda, R., De Santos, F., Lara, B., Verdugo, J., Palomera, A. y Valadez, M. (2008). Evaluación de la percepción de calidad de vida y estilo de vida en estudiantes desde el contexto de las Universidades Promotoras de la Salud. Revista de Educación y Desarrollo, 8, 5-16.

Ministerio de Salud de Perú (2016, 31 de mayo). Ambientes 100\% libres de humo de tabaco salvan vidas. Recuperado de http:// www.minsa.gob.pe/?op $=51$ ynota $=18533$.

Ministerio de Transportes y Comunicaciones (s/f). Proyecto Piloto: ;Cuida tu vida! Lima: Autor.

Ministerio de Transportes y Comunicaciones (2015). Formulación del Plan Nacional de Seguridad Vial del Perú 2015-2024. Lima: Autor. Recuperado de https://www.mtc.gob.pe/cnsv/Proyecto\%20del\%20Plan\%20Nacional\%20de\%20Seguridad\%20 Vial\%202015_2024.pdf.

Montero, A., Úbeda, M. y García, A. (2006). Evaluación de los hábitos alimenticios de una población de estudiantes universitarios en relación con sus conocimientos nutricionales. Nutrición Hospitalaria, 21(4), 466-473.

Monzón, M. (2007). Estrés académico en estudiantes universitarios. Apuntes de Psicología, 25(1), 87-99.

Oficina de las Naciones Unidades Contra la Droga y el Delito (UNODC) (2013). Abuso de drogas en adolescentes y jóvenes y vulnerabilidad familiar. Recuperado de http://www.unodc.org/documents/peruandecuador/Publicaciones/Publicaciones2014/ LIBRO_ADOLESCENTES_SPAS_UNODC-CEDRO.pdf.

Organización Mundial de la Salud (OMS) (2008). Informe de la OMS sobre la epidemia mundial de tabaquismo. Plan de medidas MPOWER. Ginebra: OMS. Recuperado de http://apps.who.int/iris/bitstream/10665/43897/1/9789243596280_spa.pdf.

Organización Mundial de la Salud (OMS) (2013). Informe OMS sobre la epidemia mundial de tabaquismo. Hacer cumplir las prohibiciones sobre publicidad, promoción y patrocinio del tabaco. Ginebra: OMS. Recuperado de http://apps.who.int/iris/ bitstream/10665/85382/1/WHO_NMH_PND_13.2_spa.pdf?ua = 1 .

Polo, A., Hernández, J. y Poza, C. (1996). Evaluación del estrés académico en estudiantes universitarios. Ansiedad y Estrés, 2(2-3), 159-172.

Pozos R., B.E., Preciado S., M., Acosta F., M., Aguilera V., M. y Delgado G., D.D. (2014). Academic stress as a predictor of chronic stress in university students. Psicología Educativa, 20, 47-54. doi: 10.1016/j.pse.2014.05.006.

Quick, V., Shoff, S., Lohnse, B., White, A., Horacek, T. y Greene, G. (2015). Relationships of eating competence, sleep behaviors and quality, and overweight status among college students. Eating Behaviors, 19, 15-19. doi: 10.1016/j.eatbeh.2015.06.012.

Roales N., J. (2004). Psicología de la salud. Aproximación histórica, conceptual y aplicaciones. Madrid: Pirámide.

Rodríguez, J. y Agulló, E. (1999). Estilos de vida, cultura, ocio y tiempo libre de los estudiantes universitarios. Psicothema, 11(2), 247-259.

Sabbah, I., Sabbah, H., Khamis, R., Sabbah, R. y Droubi, N. (2013). Health related quality of life of university students in Lebanon: Lifestyles behaviors and socio- demographic predictors. Health, 5(7), 1-12. doi: 10.4236/health.2013.57A4001.

Sajwani, R.A., Shoukat, S., Raza, R., Shiekh., M.M., Rashid, Q., Siddique, M.S., Panju, S. y Kadir, M. (2009). Knowledge and practice of healthy lifestyle and dietary habits in medical and non-medical students of Karachi, Pakistan. Journal of Pakistan Medical Association, 59(9), 650-655.

Santrock, J. (2006). Psicología del desarrollo: el ciclo vital. Madrid: McGraw-Hill/Interamericana de España, S.A.U.

Saravia, J. (2013). Factores psicológicos y conductuales de la salud en un grupo de universitarios de Lima Metropolitana. Tesis inédita de Maestría. Lima: Pontificia Universidad Católica del Perú.

Secretaría Nacional de la Juventud (SENAJU) (2012). Primera Encuesta Nacional de la Juventud. Resultados finales. Lima: Autor.

Sigmundová, D., El Ansari, W., Sigmund, E. y Frömel, K. (2011). Secular trends: a ten-year comparison of the amount and type of physical activity and inactivity of random samples of adolescents in the Czech Republic. BMC Public Health, 11(1), 731. doi: 10.1186/1471-2458-11-731.

Superintendencia de Transporte Terrestre de Personas, Carga y Mercancía (SUTRAN) (2011, 17 de junio). En 13\% disminuyeron los accidentes de tránsito con daños personales debido a planes de fiscalización ejecutados al servicio de transporte interprovincial. Lima: SUTRAN.

Taylor, S. (2007). Psicología de la salud. México: McGraw-Hill.

Thomsen, S.R. (2002). Health and beauty magazine Reading and body shape concerns among a group of college women. Journalism and Mass Comunication Quarterly, 79, 988-1007.

Vargas Z., M., Becerra B., F. y Prieto S., E. (2008). Evaluación antropométrica de estudiantes universitarios en Bogotá, Colombia. Revista de Salud Pública, 10(3), 443-452.

Vargas, P.A., Flores, M. y Robles, E. (2014). Sleep quality and body mass index in college students: the role of sleep disturbances. Journal of American College Health, 62(8), 534-541. doi: 10.1080/07448481.2014.933344.

Vella-Zarb, R.A. y Elgar, F.J. (2009). The 'Freshman 5': A meta-analysis of weight gain in the freshman year of college. Journal of American College Health, 58(2), 161-166. doi: 10.1080/07448480903221392. 
Wang, W., Kao, C., Huan, T. y Wu, C. (2011). Free time management contributes to better quality of life: a study of undergraduate students in Taiwan. Journal of Happiness Studies, 12, 561-573. doi: 10.1007/s10902-010-9217-7.

Wilson, W.D.M. y Ciliska, D. (1984). Life-style assessment: Development and use of the FANTASTIC checklist. Canadian Family Physician, 30, 1527-1532.

Yahia, N., Achkar, A., Abdallag, A. y Rizk, S. (2008). Eating habits and obesity among Lebanese university students. Nutrition Journal, 7(32), 1-6. doi: 10.1186/1475-2891-7-32.

Yahia, N., Brown, C., Rapley, M. y Chung, M. (2016). Level of nutrition knowledge and its association with fat consumption among college students. BMC Public Health, 16, 1-10. doi: 10.1186/s12889-016-3728-z. 